\title{
ĐặC ĐIỂM SỬ DỤNG AIZUCHI TRONG GIAO TIẾP NHẬTT-VIỆT XÉT TỪ QUAN ĐIỂM LÝ THUYẾT LỊCH SỰ
}

\author{
Đỗ Hoàng Ngân*
}

Khoa Ngôn ngũ và Văn hóa Nhật Bản, Trường Đại học Ngoại ngũu, ĐHQGHN, Phạm Văn Đồng, Cầu Giấy, Hà Nội, Việt Nam

Nhận bài ngày 01 tháng 12 năm 2016

Chỉnh sửa ngày 06 tháng 12 năm 2016; Chấp nhận đăng ngày 09 tháng 01 năm 2017

Tóm tắt: Nghiên cứu nhằm mục đích tìm ra những nét tương đồng và khác biệt về đặc điểm ngôn ngữ văn hóa giữa người Nhật và người Việt trong việc sử dụng aizuchi trong giao tiếp Nhật-Việt. Dữ liệu từ 12 cuộc hội thoại theo từng cặp giữa người Nhật bản ngữ với người Việt được phân tích theo các nhóm về hình thức, các nhóm chức năng của aizuchi và các nhóm về quan hệ thân sơ giữa những người tham gia hội thoại. Kết quả cho thấy, về hình thức, "Từ ngữ chêm xen" được cả người Nhật và người Việt sử dụng nhiều nhất, "Nhắc lại” được người Việt sử dụng nhiều hơn, trong khi "Diễn đạt cách khác" có tần số sử dụng cao hơn trong phát ngôn của người Nhật. Về chức năng, "Tín hiệu đang nghe" chiếm tỉ lệ cao nhất trong tổng số aizuchi của cả người Nhật và người Việt, nhưng tỉ lệ sử dụng aizuchi với chức năng này ở người Việt cao hơn người Nhật, trong khi tỉ lệ các chức năng khác ở người Nhật cao hơn người Việt, trừ “Tín hiệu phủ định" không có sự chênh lệch đáng kể. Mặt khác, nghiên cứu cũng chỉ ra rằng, aizuchi được sử dụng nhiều hơn trong giao tiếp của người Nhật bản ngữ so với người Việt bất kể mức độ quan hệ thân sơ là cao hay thấp giữa những người tham gia hội thoại. ${ }^{(1)}$

Tù̀ khóa: giao tiếp Nhật-Việt, ngôn ngữ văn hóa, giao thoa văn hóa, lý thuyết lịch sự, aizuchi

\section{Lời mở đầu}

Trong bất cứ một nền văn hóa nào, hình thức đưa ra quan điểm ý kiến của mình đều rất quan trọng, ảnh hưởng tới hiệu quả của giao tiếp nói chung. Cách thức sử dụng lời nói đóng vai trò quan trọng quyết định thành công của giao tiếp, chính vì vậy, tục ngữ Việt Nam có câu "Lời nói không mất tiền mua, lựa lời mà nói cho vừa lòng nhau". Việc sử dụng lời nói có thể gây ra những hậu quả khó lường đã được đúc kết trong kho tàng tục ngữ của nhiều dân tộc. Tục ngữ Nhật Bản có câu: “口は災いのもと” (Kuchi wa wazawai no moto: Lời nói (miệng) là nguồn gốc của thảm họa), trong khi tục ngữ Việt Nam nói: "Không có cái gì độc bằng cái lưỡi".

* ĐT.: 84-942969309, E-mail: dhnganhn@gmail.com

${ }^{1}$ Nghiên cứu này được hoàn thành với sự hỗ trợ của Đại học Quốc gia Hà Nội trong đề tài mã số QG.14.50
Trong thế giới ngày nay, những tình huống giao tiếp trong đó người tham gia đến từ các nền văn hóa khác nhau đã trở nên phổ biến. Những năm gần đây, cùng với sự phát triển mạnh mẽ và sâu rộng của quan hệ NhậtViệt, các cơ hội giao lưu Nhật-Việt không còn là những tình huống hiếm gặp. Người Nhật vốn được biết đến trên toàn thế giới là một dân tộc lịch sự và có nhiều nghi lễ trong giao tiếp. Một trong những biểu hiện rõ nét văn hóa Nhật trong giao tiếp chính là cách sử dụng kính ngữ, một vấn đề được coi là khó đối với những người nước ngoài học tiếng Nhật, thậm chí cả đối với nhiều người Nhật, nhất là thế hệ trẻ. Trong tiếng Nhật, ngoài các cấu trúc và cách diễn đạt tôn kính với nhiều hình thức thể hiện các mức độ khác nhau, còn có các cấu trúc và cách diễn đạt khiêm nhường dùng để 
nói về bản thân. Để có thể tham gia giao tiếp thành công và tự nhiên bằng tiếng Nhật, không thể không bàn đến một phạm trù liên quan đến chuẩn mực lịch sự của người Nhật và không thể thiếu được trong giao tiếp là aizuchi trong tiếng Nhật. Việc nghiên cứu, tìm hiểu chúng một cách hệ thống, cụ thể trong giao tiếp là hết sức cần thiết và hữu ích. Cho đến nay, đã có một số nghiên cứu về aizuchi trong tiếng Nhật, song chưa có nghiên cứu nào tìm hiểu làm sáng tỏ về đặc điểm sử dụng aizuchi của người Nhật và người Việt trong giao tiếp Nhật Việt. Chính vì vậy, đây là mục tiêu mà chúng tôi đặt ra cho nghiên cứu này.

Kĩ năng giao tiếp bao gồm cả các qui tắc giao tiếp ngôn từ và các qui tắc giao tiếp phi ngôn từ, chúng được qui định và đánh giá theo các chuẩn mực văn hóa chung và của mỗi cộng đồng xã hội. Trong bài viết này chúng tôi sẽ đề cập đến đặc điểm giao tiếp ngôn từ thông qua phân tích việc sử dụng aizuchi của người Nhật và người Việt trong giao tiếp Nhật-Việt.

\section{Lý thuyết lịch sự và văn hóa giao tiếp của người Nhật}

Trên thế giới, lý thuyết về các vấn đề giao tiếp, trong đó có lý thuyết lịch sự đã được nhiều học giả quan tâm nghiên cứu từ khá lâu (Lakoff, 1977; Leech, 1983, Brown \& Levinson, 1987; Grice, 1997; v.v.). Các nhà nghiên cứu đã tìm hiểu, phân tách các yếu tố và xem xét chúng trong các điều kiện bối cảnh cụ thể của quá trình giao tiếp, xác định mối quan hệ và tác động chi phối của các yếu tố nội tại cũng như ngoại vi trong giao tiếp.

Để đạt mục đích giao tiếp, để người nghe lĩnh hội được những điều mà người nói muốn truyền đạt, phát ngôn nêu ra phải thỏa mãn những điều kiện nhất định được qui định bởi bối cảnh và mối quan hệ giữa người nói và người nghe. Grice (1997) đã đưa ra Nguyên tắc hợp tác (Co-operative Principle) với 4 phương châm. Một là Phương châm về lượng (Maxim of Quantity): làm sao cho lượng thông tin đưa ra vừa đủ với mục đích của cuộc hội thoại. Hai là Phương châm về chất (Maxim of Quality): cố gắng đưa thông tin đúng, đặc biệt là không nói những điều mà bản thân mình tin là sai và không nói những điều thiếu bằng chứng. $\mathrm{Ba}$ là Phương châm về sự liên quan (Maxim of Relation): làm cho phần tham gia của mình phù hợp. Bốn là Phương châm về cách thức (Maxim of Manner): diễn đạt rõ ràng, cụ thể là tránh thể hiện tối nghĩa, tránh mập mờ, nói ngắn gọn và nói có trình tự. Những phương châm trên đây không phải luôn được nêu ra thành lời, nhưng là những ý niệm chung, những qui tắc chung mà nếu người tham gia hội thoại vi phạm thì có thể gây khó khăn cho giao tiếp.

Tuy nhiên, trong giao tiếp, không phải bao giờ chúng ta cũng có thể nói được hoặc cũng cần nói thẳng ra tất cả những điều muốn nói. Cùng với Nguyên tắc hợp tác, Nguyên tắc lịch sự được coi là nguyên tắc quan trọng, làm nền tảng cho quá trình giao tiếp, là chuẩn mực văn hóa được qui định bởi mỗi cộng đồng xã hội. Leech (1983) đã đưa ra 6 phương châm trong Nguyên tắc lịch sự. Một là Phương châm tế nhị (Tact Maxim): giảm tối đa cái thiệt, tăng tối đa cái lợi cho người khác. Hai là Phương châm quảng đại (Generosity Maxim): giảm tối đa cái lợi, tăng tối đa cái thiệt về mình. Ba là Phương châm tán thưởng (Approbation Maxim): giảm tối đa việc phê phán, tăng tối đa việc tán thưởng với người khác. Bốn là Phương châm khiêm tốn (Modesty Maxim): giảm tối đa việc tán thưởng, tăng tối đa việc phê phán đối với bản thân. Năm là Phương châm đồng thuận (Agreement Maxim): giảm 
tối đa sự bất đồng, tăng tối đa sự thống nhất ý kiến với người khác. Sáu là Phương châm cảm thông (Sympathy Maxim): giảm tối đa sự không đồng cảm, tăng tối đa sự đồng cảm với người khác.

Một trong những phạm trù được nhiều nhà nghiên cứu coi là phạm trù cơ bản và quan trọng của nguyên tắc lịch sự trong giao tiếp là Thể diện (Face). Brown và Levinson (1987) sử dụng khái niệm thể diện được Gofman (1967) đưa ra và xây dựng mô hình giao tiếp với các hành động giao tiếp tuân thủ theo những qui tắc nhằm bảo vệ thể diện của đối tác. Brown và Levinson đã đưa ra công thức tính toán mức độ đe dọa thể diện: $\mathrm{Wx}=$ $\mathrm{DS}, \mathrm{H}+\mathrm{PH}, \mathrm{S}+\mathrm{RX}$, trong đó $\mathrm{Wx}$ là mức độ đe dọa thể diện, $\mathrm{DS}, \mathrm{H}$ là khoảng cách xã hội hay quan hệ thân sơ giữa người nói và người nghe, $\mathrm{PH}, \mathrm{S}$ là quyền lực giữa người nghe đối với người nói, $\mathrm{RX}$ là mức độ áp đặt của hành vi ngôn ngữ trong nền văn hóa của người nói và người nghe.

Thomas Holtgraves (2001) cũng cho rằng việc sử dụng ngôn ngữ hiệu quả đòi hỏi sự hợp tác, và việc đáp ứng yêu cầu này chính là thực tế rằng mọi người vừa là người nói đồng thời là người nghe. Người nói đưa ra các phát ngôn nhằm mục đích cho ý định của mình được hiểu, và người tiếp nhận xử lí những nhận xét của người nói với mục tiêu công nhận những điều đó. Thomas cho rằng tất cả mọi người tham gia giao tiếp phải làm sao để tránh làm mất lòng nhau.

Yamada (1997) đã ví giao tiếp giao văn hóa (cross-cultural communication) như sự tiếp xúc của những người chơi các trò chơi khác nhau. Mỗi người chơi đều mong chờ và cho rằng cuộc chơi sẽ diễn ra theo các qui tắc chơi của họ. Song giống như việc bạn không thể chơi bóng chày mà áp dụng các qui tắc của bóng đá Mỹ, bạn cũng không thể tham gia giao tiếp ở Mỹ mà sử dụng các qui tắc của Nhật Bản và ngược lại.

Ở Việt Nam, những vấn đề về giao tiếp giao văn hóa đã được nhiều nhà nghiên cứu, nhiều người làm công tác giảng dạy ngoại ngũ̃, làm về lĩnh vực đối ngoại, ngoại giao quan tâm và đề cập đến từ khá lâu. Giao tiếp giao văn hóa đã được nghiên cứu với các cách tiếp cận khác nhau từ những vấn đề lý luận chung như thái độ giao tiếp, quan hệ giao tiếp, tới những nghiên cứu các phương diện cụ thể như ngữ âm, cách sử dụng từ ngữ trong giao tiếp, hay các hành động ngôn từ cụ thể như cách thức khen, cách từ chối lời mời. Nguyễn Quang (2002) đã đề cập một cách hệ thống đến nhiều bình diện của quá trình giao tiếp giao văn hóa như "Chủ quan tính - Khách quan tính", "Trực tiếp - Gián tiếp - Lịch sự”, cùng những nghiên cứu thực nghiệm về hành động ngôn trung giao tiếp cụ thể như "Khen trong dụng học giao văn hóa Việt - Mỹ) và nghiên cứu thực nghiệm về giao tiếp phi ngôn từ.

Nghiên cứu về giao tiếp giao văn hóa NhậtViệt, Hoàng Anh Thi $(1997,2001)$ khảo sát từ ngữ xưng hô và hoạt động của chúng trong hai ngôn ngữ Nhật-Việt nhằm đưa ra những điểm tương đồng và khác biệt trong cấu trúc hệ thống và trong nguyên lí hoạt động của các phương tiện xưng hô của hai ngôn ngữ này, đồng thời lí giải những nét tương đồng và khác biệt đó bằng các đặc trưng văn hóa, tâm lí và xã hội.

\section{Aizuchi trong giao tiếp của người Nhật}

Aizuchi đã được đề cập đến trong nhiều công trình như 大宮 (1986), 水谷 (1988), メイナード (1993), 堀口 (1997), 村田 (2000), 大塚 (2007, 2009). Cho đến nay, các nhà nghiên cứu đã đưa ra một số cách định 
nghĩa khác nhau về aizuchi trong tiếng Nhật (aizuchi). Aizuchi tiếng Nhật, tiếng Anh là "back-channel" tạm dịch là "kênh phản hồi". Trong tiếng Việt hiện nay không có một từ tương đương với aizuchi trong tiếng Nhật. Aizuchi hiểu theo nghĩa hẹp, tương đương với aizuchi shi, trong Từ điển Nhật-Việt( ${ }^{(2)}$ không có “あいづち” như một từ riêng, mà xuất hiện trong cụm từ “あいづちをうつ” nghĩa là phụ họa theo, nói dựa theo ${ }^{(3)}$. Aizuchi theo nghĩa rộng, có thể hiểu tương đương với "phát ngôn chêm xen" trong tiếng Việt, trong đó bao gồm cả "từ ngữ chêm xen", câu nhắc lại, diễn đạt cách khác cũng như từ ngữ chêm xen đi trước hay phát ngôn chêm xen đi trước. Theo chúng tôi, định nghĩa aizuchi của 堀口 (1997: 42) là đầy đủ và khái quát, vì vậy, trong nghiên cứu này chúng tôi lấy đó làm căn cứ để xác định và phân tích. "Đó là những phát ngôn được đưa ra trong khi người nói đang thực hiện quyền phát ngôn của mình, thể hiện những điểm chung từ thông tin mà người nói đưa ra". Như vậy, chúng tôi xem xét aizuchi theo nghĩa rộng, không chỉ hạn chế là các từ ngữ chêm xen như え, うん, そう, はい, あのう， え え, はい, いや，さあ，あっ，ええと，ま あ, ねえ, まあね, じやあ, あら, へえ, なるほど，そうか，いいなあ，ほうんと うだ, mà bao gồm cả những phát ngôn như nhắc lại, diễn đạt lại một phát ngôn bằng cách khác, hoặc những từ ngữ, phát ngôn mà người nghe phán đoán trước nội dung của người nói và chêm xen vào khi đang nghe giữa chừng.

Aizuchi là một bộ phận quan trọng trong ngôn ngữ Nhật Bản. Tần số sử dụng aizuchi

${ }^{2}$ Lê Đức Niệm và tập thể tác giả, Tù điển Nhật - Việt, NXB. Mũi Cà Mau, 1994, tr. 9.

3 Trong từ điển còn đưa nghĩa thứ 2 là "luân đánh búa", không liên quan đến giao tiếp nên chúng tôi không đề cập ở nghiên cứu này. trong giao tiếp tiếng Nhật được khảo sát trong khá nhiều nghiên cứu như 水谷 (1984), 小宮 (1986), 黒崎 (1987), 大塚 (2005). Trong khi 水谷 (1984) tính tần số theo âm tiết trong lời nói thì 小宮 (1986) tính theo đơn vị thời gian là giây, 黒崎 (1987) và 大塚 (2005) tính tỉ lệ câu chứa aizuchi trên tổng số phát ngôn.

Về chức năng của aizuchi trong tiếng Nhật, các nhà nghiên cứu có những cách phân loại khác nhau. メイナード (1993) đưa ra 6 nhóm là (1) Tín hiệu hãy tiếp tục, (2) Tín hiệu hiểu nội dung, (3) Tín hiệu ủng hộ phán đoán của người nói, (4) Tín hiệu tán thành cách nghĩ, ý kiến của đối tác, (5) Tín hiệu mạnh mẽ biểu đạt tình cảm và (6) Biểu đạt yêu cầu, chỉnh sửa, bổ sung thông tin. 堀口 (1997) chia ra 5 nhóm chức năng là (1) Tín hiệu đang nghe, (2) Tín hiệu hiểu, (3) Tín hiệu đồng tình, (4) Tín hiệu phủ định và (5) Biểu đạt tình thái. 楊 (2001) chia các aizuchi thành 4 loại là (1) Tín hiệu đang nghe, (2) Tín hiệu hiểu, (3) Biểu đạt sự đồng ý, đồng cảm và (4) Biểu đạt tình thái. Chúng tôi thấy cách phân loại của堀 口 (1997) khá rõ ràng, đầy đủ, không có nhiều trường hợp khó phân định, vì vậy, trong bài viết này chúng tôi căn cứ vào cách phân loại về chức năng đó để phân tích, xem xét các aizuchi trong giao tiếp Nhật-Việt.

Về hình thức của aizuchi, theo堀口 (1997), ngoài từ ngữ chêm xen, bao gồm cả nhắc lại, diễn đạt cách khác, từ ngữ chêm xen đi trước và phát ngôn chêm xen đi trước.

Về thời điểm aizuchi được đưa ra, nhiều nhà nghiên cứu đã phân tích và xác định thời điểm aizuchi thường được đưa ra là sau trợ từ 「ね」và thời gian dừng nghỉ (小宮, 1986), hay là khi đáp lại những câu có trợ từ cuối câu 「なあ」「のう」, những chỗ được thể hiện bằng giọng nhẹ đi (黒崎, 1987). 
Trong các nghiên cứu về ngôn ngữ Nhật Bản và giáo dục tiếng Nhật, đã có khá nhiều nghiên cứu về aizuchi như một bộ phận không thể thiếu trong tiếng Nhật, đặc biệt là khi phương pháp phân tích diễn ngôn phát triển và trở thành một thủ pháp quan trọng trong nghiên cứu liên quan đến giao tiếp. Trong đó, bao gồm cả các nghiên cứu về bình diện ngôn ngữ học cũng như về mặt thực tiễn sử dụng chúng trong giao tiếp, hay nghiên cứu đối chiếu tiếng Nhật và các thứ tiếng khác. Có một số nghiên cứu đối chiếu tần số sử dụng aizuchi trong giao tiếp của người Nhật bản ngữ và trong giao tiếp của người có tiếng mẹ đẻ là các thứ tiếng khác, song nhiều nhất là với các thứ tiếng như tiếng Trung, tiếng Hàn, tiếng Anh như nghiên cứu của LoCastro (1987), White (1989), 堀口 (1990), 楊 (1997) và Mukai (1999). Tùy vào mục đích nghiên cứu mà đối tượng tham gia hội thoại trong các nghiên cứu có những tiêu chí khác nhau. Với mục đích tìm hiểu xem có sự khác nhau không giữa người bản ngữ và phi bản ngữ, có sự ảnh hưởng của tiếng mẹ đẻ tới việc sử dụng aizuchi trong giao tiếp của người phi bản ngữ không, 楊 (1997) đã so sánh hội thoại giữa những người Nhật bản ngữ (JJ), hội thoại giữa những người Trung Quốc $(\mathrm{CC})$ và hội thoại giữa người Trung Quốc với người Nhật bản ngữ. Kết quả chỉ ra là $\mathrm{JJ}>\mathrm{CJ}>\mathrm{CC}$ và tiếng mẹ đẻ có ảnh hưởng tới hình thức sử dụng aizuchi trong giao tiếp. Mukai (1999) so sánh hội thoại của 5 cặp người Nhật với nhau và 5 cặp giữa người Nhật với người học tiếng Nhật ở trình độ cao cấp mà tiếng mẹ đẻ là tiếng Anh. Kết quả cho thấy người học tiếng Nhật ở trình độ cao có tần số sử dụng aizuchi không khác mấy so với người Nhật bản ngữ.

Như trên chúng tôi đã trình bày, trong các nghiên cứu về ngôn ngữ Nhật Bản và nghiên cứu giáo dục tiếng Nhật, đã có khá nhiều nghiên cứu về các bình diện ngôn ngữ học cũng như thực tiễn sử dụng chúng trong giao tiếp, tuy nhiên, cho đến nay chưa có nghiên cứu nào đi sâu so sánh đặc điểm sử dụng aizuchi của người Nhật và người Việt trong giao tiếp Nhật-Việt. Trong nghiên cứu này, để phân tích, xem xét các aizuchi trong giao tiếp Nhật-Việt, chúng tôi sẽ phân chia các aizuchi trong tiếng Nhật theo 5 nhóm về hình thức là (1) Từ ngữ chêm xen, (2) Nhắc lại, (3) Diễn đạt cách khác, (4) Từ ngữ chêm xen đi trước và (5) Phát ngôn chêm xen đi trước; 5 nhóm theo chức năng là (1) Tín hiệu đang nghe, (2) Tín hiệu hiểu, (3) Tín hiệu đồng tình, (4) Tín hiệu phủ định và (5) Biểu đạt tình thái.

Cùng với hoạt động lời nói, các yếu tố phi ngôn từ cũng đóng một vai trò hết sức quan trọng trong giao tiếp, đặc biệt là giao tiếp trực diện. Nhật Bản là một dân tộc có nhiều nguyên tắc khá nghiêm ngặt và tỉ mỉ về các hành vi giao tiếp, bao gồm cả giao tiếp ngôn từ và giao tiếp phi ngôn từ. Tuy nhiên, trong bài viết này chúng tôi không đề cập đến các yếu tố phi ngôn từ, mà tập trung vào quan sát giao tiếp ngôn từ và phân tích các đặc điểm của chúng.

\section{Khảo sát về việc sử dụng aizuchi trong giao tiếp Nhật-Việt}

\subsection{Mục đích của khảo sát}

Khảo sát được tiến hành nhằm làm rõ những điểm sau đây về việc sử dụng aizuchi trong giao tiếp Nhật-Việt:

1) Tần số sử dụng aizuchi của người Nhật và người Việt giống hay khác nhau?

2) Các loại aizuchi mà người Nhật và người Việt sử dụng giống hay khác nhau?

3) Chức năng của aizuchi mà người Nhật và người Việt sử dụng giống hay khác nhau? 
4) Yếu tố khoảng cách xã hội (quan hệ thân sơ) có ảnh hưởng tới việc sử dụng aizuchi trong giao tiếp không?

\section{2. Đối tượng tham gia khảo sát}

Đối tượng tham gia khảo sát này bao gồm 12 người Nhật được kí hiệu từ $\mathrm{J} 1$ đến $\mathrm{J} 12$ và 12 người Việt được kí hiệu từ V1 đến V12, trong đó số lượng nam và nữ tương ứng đều bằng nhau là 6 nam (các kí hiệu có số lẻ) và 6 nữ (các kí hiệu có số chẵn). Người tham gia khảo sát V1 đến V12 có trình độ tiếng Nhật N4 là 2 người, N3 là 5 người, N2 là 4 người và N1 là 1 người.

Độ tuổi của những người tham gia khảo sát là từ 23 đến 52 tuổi.

\subsection{Phuoong pháp tiến hành khảo sát}

Những phương pháp chủ yếu được sử dụng trong nghiên cứu là điều tra khảo sát, phân tích - tổng hợp, thống kê định lượng. Chúng tôi cũng sử dụng phương pháp so sánh đối chiếu để tìm ra những điểm khác biệt giữa các nhóm đối tượng được khảo sát.

Hội thoại được thực hiện theo từng cặp giữa một người Nhật và một người Việt. Những người tham gia khảo sát được gợi ý nói chuyện về các chủ đề thông dụng của cuộc sống hàng ngày như việc học ngoại ngữ, các hoạt động giải trí và sở thích. Thời gian của mỗi cuộc hội thoại là từ 15 đến 25 phút.

Dữ liệu được thu thập bằng hình thức ghi âm các cuộc hội thoại sử dụng điện thoại thông minh. Sau đó, các cuộc hội thoại được văn bản hóa để thống kê, phân tích.

Sau khi kết thúc hội thoại, những người tham gia trả lời một số câu hỏi xung quanh việc giao tiếp với người cùng hội thoại, việc sử dụng aizuchi và ý thức về việc sử dụng aizuchi của bản thân mình và của người cùng tham gia hội thoại.

\subsection{Thời gian khảo sát}

Khảo sát được thực hiện trong thời gian từ tháng 7.2016 đến tháng 9.2016.

\subsection{Kết quả khảo sát}

\subsubsection{Tần số sử dụng aizuchi}

Thống kê số liệu đã được văn bản hóa của từng hội thoại cho kết quả cụ thể như sau:

Bảng 1. Tần số sử dụng aizuchi của người Nhật và người Việt trong giao tiếp Nhật-Việt

\begin{tabular}{|c|c|c|c|c|}
\hline Hội thoại & $\begin{array}{l}\text { Người } \\
\text { phát } \\
\text { ngôn }\end{array}$ & $\begin{array}{c}\text { Tổng số } \\
\text { phát } \\
\text { ngôn }\end{array}$ & $\begin{array}{c}\text { Số } \\
\text { aizuchi }\end{array}$ & $\begin{array}{l}\text { Tỉ lệ aizuchi } \\
\text { trên tổng số } \\
\text { phát ngôn }\end{array}$ \\
\hline \multirow{2}{*}{$\begin{array}{c}\text { Hội thoại } 1 \\
\text { (K1) }\end{array}$} & J1 & 298 & 66 & $22 \%$ \\
\hline & V1 & 255 & 26 & $10 \%$ \\
\hline \multirow{2}{*}{$\begin{array}{c}\text { Hội thoại } 2 \\
\text { (K2) }\end{array}$} & $\mathrm{J} 2$ & 451 & 192 & $43 \%$ \\
\hline & $\mathrm{V} 2$ & 265 & 41 & $15 \%$ \\
\hline \multirow{2}{*}{$\begin{array}{c}\text { Hội thoại } 3 \\
\text { (K3) }\end{array}$} & $\mathrm{J} 3$ & 348 & 59 & $17 \%$ \\
\hline & V3 & 360 & 79 & $22 \%$ \\
\hline \multirow{2}{*}{$\begin{array}{l}\text { Hội thoại } 4 \\
\text { (K4) }\end{array}$} & $\mathrm{J} 4$ & 374 & 109 & $29 \%$ \\
\hline & V4 & 258 & 35 & $14 \%$ \\
\hline \multirow{2}{*}{$\begin{array}{l}\text { Hội thoại } 5 \\
\text { (K5) }\end{array}$} & J5 & 389 & 138 & $35 \%$ \\
\hline & V5 & 371 & 102 & $27 \%$ \\
\hline \multirow{2}{*}{$\begin{array}{c}\text { Hội thoại } 6 \\
\text { (K6) }\end{array}$} & J6 & 285 & 117 & $41 \%$ \\
\hline & V6 & 247 & 94 & $38 \%$ \\
\hline \multirow{2}{*}{$\begin{array}{c}\text { Hội thoại } 7 \\
\text { (K7) }\end{array}$} & $\mathrm{J} 7$ & 358 & 104 & $29 \%$ \\
\hline & V7 & 296 & 48 & $16 \%$ \\
\hline \multirow{2}{*}{$\begin{array}{l}\text { Hội thoại } 8 \\
\text { (K8) }\end{array}$} & J8 & 335 & 161 & $48 \%$ \\
\hline & V8 & 241 & 65 & $27 \%$ \\
\hline \multirow{2}{*}{$\begin{array}{l}\text { Hội thoại } 9 \\
\text { (K9) }\end{array}$} & J9 & 320 & 150 & $47 \%$ \\
\hline & V9 & 180 & 19 & $11 \%$ \\
\hline \multirow{2}{*}{$\begin{array}{l}\text { Hội thoại } 10 \\
\text { (K10) }\end{array}$} & $\mathrm{J} 10$ & 360 & 166 & $46 \%$ \\
\hline & V10 & 329 & 122 & $37 \%$ \\
\hline \multirow{2}{*}{$\begin{array}{l}\text { Hội thoại } 11 \\
\text { (K11) }\end{array}$} & J11 & 297 & 72 & $24 \%$ \\
\hline & V11 & 325 & 97 & $30 \%$ \\
\hline \multirow{2}{*}{$\begin{array}{l}\text { Hội thoại } 12 \\
\text { (K12) }\end{array}$} & $\mathrm{J} 12$ & 334 & 152 & $46 \%$ \\
\hline & V12 & 234 & 91 & $39 \%$ \\
\hline
\end{tabular}

Kết quả tổng hợp 12 hội thoại J1 J12 như sau: tổng số aizuchi là 1486 trên tổng số toàn bộ 4149 phát ngôn, chiếm 35,8\%. Trong khi V1 V12 có tổng số aizuchi là 819 trên tổng số toàn bộ 3361 phát ngôn, chiếm 24,4\%. 


\subsubsection{Các loại aizuchi được sủ dụng}

Như trên đã trình bày, theo cách phân loại các aizuchi của 堀口 (1997), chúng tôi thống kê số lượng aizuchi theo 5 nhóm và kết quả cụ thể trong bảng 2 .

Trong đó, những aizuchi thường gặp nhiều nhất là từ ngữ chêm xen $(\mathrm{P} 1)$ như うん, え, ええ, そう,はい,いや，さあ，あっ，あのう，え えと，まあ，ねえままね，じやあ，あ ら，へえ，なるほど，そうか，そうなん だ，いいなあ，ほんとう，ほうんとうだ。

Ví dụ:

(K7) J1：うん、他の学生に頼んでみ たらいんじゃないか。

V1: いや.。。それはちょっ とまずいなあ。

(K4)V4: 旅行がすきですか。

J4: え、好きですよ。

V4: え、国内旅行、え、外国の旅 行どちらのほうが好きですか。

J4: まあ、旅行が好きですけど、 やっぱり、お金があるなら、海外に行き たいです。

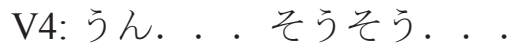

J4: はい。 .

(K11) : V11：その人のすることには 口を出さない

$\mathrm{J} 11$ ：そうそう。.

V11：ということがなかなか出来 ないんですね。

J11：そうですか

Bảng 2. Kết quả thống kê các aizuchi theo nhóm hình thức

\begin{tabular}{|l|c|c|c|c|}
\hline & \multicolumn{2}{|c|}{ J } & \multicolumn{2}{c|}{ V } \\
\hline Từ ngữ chêm xen (P1) & 1158 & $77,9 \%$ & 662 & $80,8 \%$ \\
\hline Nhắc lại (P2) & 98 & $6,6 \%$ & 76 & $9,3 \%$ \\
\hline Diễn đạt cách khác (P3) & 176 & $11,9 \%$ & 52 & $6,3 \%$ \\
\hline Từ ngữ chêm xen đi trước (P4) & 42 & $2,8 \%$ & 26 & $3,2 \%$ \\
\hline $\begin{array}{l}\text { Phát ngôn chêm xen đi } \\
\text { trước (P5) }\end{array}$ & 12 & $0,8 \%$ & 3 & $0,4 \%$ \\
\hline Tổng cộng & 1486 & $100 \%$ & 819 & $100 \%$ \\
\hline
\end{tabular}

Ngoài từ ngữ chêm xen, trong hội thoại, có những phát ngôn khác cũng được đưa ra mà không phải để thực hiện quyền phát ngôn của mình, chúng bao gồm cả chức năng xác nhận việc có chung thông tin. Đó là những phát ngôn như nhắc lại nguyên văn lời người cùng đối thoại (P2), diễn đạt lại một phần hoặc toàn bộ nội dung của một phát ngôn bằng cách khác (P3), hay từ ngữ chêm xen hoặc phát ngôn chêm xen được đưa ra trước câu trả lời khi người nghe dự đoán trước nội dung phát ngôn của người đối thoại (P4, P5).

Ví dụ:

(K8)：J8：この寒さ。. .もし暖房 がなかったら

V8:暖房 . . 暖房?

J8: え... クーラーじゃなくて... $[P 3]$

V8：あつ。. . 暖房だよね。そう だよねえ。。 。

J8：ねえ。。. 。

(K11): V11：最近. .との 良いこと は. .

$\mathrm{J} 11:$ そうですね。[P4]

V11：読書 やっぱり魅力的です ねえ。

J11：はい. 本当ですね。

(K12): V11:水泳好きな人がですね、 あのう。. . 雨の中でも水泳をやるよう にですねえ。スキーが好きな人というの は. .

J11：ええ。. それはそうかも しれませんね。 [P5]

V11：どんな天気でもしたい気持 ちがあるんから ...

J11： そうですね。

V11: 㸚え...

4.5.3. Kết quả về việc sủ dụng aizuchi theo nhóm chức năng 
Theo cách phân loại aizuchi của 堀口 (1997), chúng tôi thống kê số lượng aizuchi theo 5 nhóm chức năng và kết quả cụ thể như sau:

Bảng 3. Kết quả thống kê aizuchi theo chức năng

\begin{tabular}{|l|c|c|c|c|}
\hline & \multicolumn{2}{|c|}{ J } & \multicolumn{2}{|c|}{ V } \\
\hline Tín hiệu đang nghe (A) & 631 & $42,5 \%$ & 482 & $58,9 \%$ \\
\hline Tín hiệu hiểu (B) & 185 & $12,4 \%$ & 66 & $8,0 \%$ \\
\hline Tín hiệu đồng tình (C) & 282 & $19,0 \%$ & 93 & $11,4 \%$ \\
\hline Tín hiệu phủ định (D) & 80 & $5,4 \%$ & 48 & $5,8 \%$ \\
\hline Biểu đạt tình thái (E) & 308 & $20,7 \%$ & 130 & $15,9 \%$ \\
\hline Tồng cộng & 1486 & $100 \%$ & 819 & $100 \%$ \\
\hline
\end{tabular}

Trong đó, ở cả người Nhật bản ngữ và người Việt, các aizuchi thường gặp nhiều nhất là với chức năng (A) Tín hiệu đang nghe, chiếm $42,5 \%$ trong tổng số aizuchi của người Nhật và $58,9 \%$ trong tổng số aizuchi của người Việt. Những aizuchi với chức năng (A) thường gặp là う，うう， え， ええ，は い，うん，そうか，そうなんだ。

Tiếp sau đó là chức năng (E) Biểu đạt tình thái chiếm $20,7 \%$ trong tổng số aizuchi của người Nhật và $15,9 \%$ trong tổng số aizuchi của người Việt. Những aizuchi với chức năng (E) thường gặp là えつ, あつ, ほんと う，あれ， へえ，いいなあ。

Aizuchi với chức năng (B) Tín hiệu hiểu, chiếm $12,4 \%$ trong tổng số aizuchi của người Nhật và $8 \%$ trong tổng số aizuchi của người Việt. Những aizuchi với chức năng $(\mathrm{B})$ thường gặp là はい, わかった, うん, う んうん.

Aizuchi với chức năng $(\mathrm{C})$ Tín hiệu đồng tình chiếm 19\% trong tổng số aizuchi của người Nhật và $11,4 \%$ trong tổng số aizuchi của người Việt. Những aizuchi với chức năng (C) thường gặp là そう，そうそう，ほんと うだ，䘛え，そうね。

Tần số xuất hiện aizuchi với chức năng (D) Tín hiệu phủ định thấp nhất ở cả nhóm người Nhật và người Việt, chỉ chiếm $5,4 \%$ trong tổng số aizuchi của người Nhật và $5,8 \%$ trong tổng số aizuchi của người Việt. Những aizuchi với chức năng $(\mathrm{D})$ thường gặp là $\quad$ W や, いやいや, いえいえ.

\subsubsection{Kết quả thống kê aizuchi theo mưc} độ thân so

Dữ liệu các cuộc hội thoại được chia thành hai nhóm, căn cứ vào thời gian quen biết, số lần giao tiếp thông qua tất cả các hình thức, theo sự đánh giá của bản thân những người tham gia về mức độ thân sơ với người cùng tham gia hội thoại, chúng tôi chia các cuộc hội thoại thành hai nhóm theo mức độ thân sơ. Hội thoại 1 đến Hội thoại 6 là những hội thoại mà những người tham gia có mức độ thân sơ cao, gọi là nhóm DH. Hội thoại 7 đến Hội thoại 12 là những hội thoại mà những người tham gia có mức độ thân sơ thấp, gọi là nhóm DL. Kết quả thống kê tần số được sử dụng của từng loại aizuchi trong hội thoại của hai nhóm DH và DL được thể hiện ở bảng sau:

Bảng 4. Kết quả thống kê aizuchi theo nhóm $\mathrm{DH}$ và $\mathrm{DL}$

\begin{tabular}{|l|c|c|c|c|c|c|}
\hline \multirow{2}{*}{} & \multicolumn{3}{|c|}{ Nhóm DH } & \multicolumn{3}{c|}{ Nhóm DL } \\
\cline { 2 - 7 } & $\mathrm{J}$ & $\mathrm{V}$ & $\begin{array}{c}\text { Tổng } \\
\text { cộng }\end{array}$ & $\mathrm{J}$ & $\mathrm{V}$ & $\begin{array}{c}\text { Tổng } \\
\text { cộng }\end{array}$ \\
\hline $\begin{array}{l}\text { Số } \\
\text { aizuchi }\end{array}$ & 681 & 377 & 1058 & 805 & 442 & 1247 \\
\hline $\begin{array}{l}\text { Tồng số } \\
\text { phát ngôn }\end{array}$ & 2145 & 1756 & 3901 & 2004 & 1605 & 3609 \\
\hline $\begin{array}{l}\text { Tỉ lệ số } \\
\text { aizuchi/ } \\
\text { Tống số } \\
\text { phát ngôn }\end{array}$ & $31,7 \%$ & $21,5 \%$ & $27,1 \%$ & $40,1 \%$ & $27,5 \%$ & $34,6 \%$ \\
\hline
\end{tabular}

\section{Quan sát}

Trên đây chúng tôi đã trình bày kết quả về việc sử dụng aizuchi qua các cuộc hội thoại theo từng cặp giữa người Nhật bản ngữ và người Việt có trình độ từ N3 đến N1. Dữ liệu được phân chia theo 5 nhóm hình thức, 5 nhóm chức năng và 2 nhóm theo mức độ 
thân sơ. Từ các kết quả trên chúng tôi thấy một số đặc điểm sau về việc sử dụng aizuchi trong giao tiếp Nhật-Việt của người Nhật và người Việt.

\subsection{Tần số sủ dụng aizuchi trong giao tiếp} Nhật-Việt

Trong bất cứ một nền văn hóa nào, hình thức đưa ra quan điểm ý kiến của mình đều rất quan trọng, quyết định thành công của giao tiếp nói chung. Trong quá trình giao tiếp, không phải bao giờ người nói cũng nêu ra tất cả mọi điều hay thể hiện được mọi điều mà mình muốn biểu đạt. Ví dụ, việc phủ nhận hay từ chối thẳng, một cách đột ngột ý kiến, quan điểm của người cùng tham gia hội thoại mà không giải thích gì thường là cách giao tiếp khó được chấp nhận trong mọi nền văn hóa, mọi ngôn ngữ. Tuy nhiên, ở mỗi nền văn hóa, mỗi ngôn ngữ, cách diễn đạt có những mức độ trực tiếp hay gián tiếp khác nhau. Aizuchi là một trong những phương tiện đóng vai trò quan trọng giúp người tham gia giao tiếp điều chỉnh cho hội thoại trở nên tự nhiên, mềm mại hơn, giống người bản ngữ hơn và giúp thể hiện sự quan tâm của người nghe đối với phát ngôn mà người nói đưa ra. Kết quả thống kê (Bảng 1) cho thấy tổng số aizuchi xuất hiện trong phát ngôn của người Nhật ở tất cả các hội thoại dữ liệu là 1486, cao hơn đáng kể so với tổng số aizuchi xuất hiện trong phát ngôn của người Việt là 819. So sánh tần số aizuchi xuất hiện trong phát ngôn của người Nhật và người Việt ở từng hội thoại, chúng tôi thấy, ở phần lớn các hội thoại, đó là K1, K2, K4, K5, K6, K7, K8, $\mathrm{K} 9, \mathrm{~K} 10, \mathrm{~K} 12$, tần số sử dụng aizuchi của người Nhật cao hơn so với người Việt. Chỉ riêng ở K3 và K11, tần số sử dụng aizuchi của người Việt cao hơn so với người Nhật song sự chênh lệch không lớn. Kết quả này phù hợp với kết quả nghiên cứu trước đây về tần số sử dụng aizuchi giữa người Nhật bản ngữ và phi bản ngữ của 楊 (1997) và Mukai (1999).

Quan sát tất cả các cuộc hội thoại trong khảo sát này, chúng tôi nhận thấy rằng người Nhật rất chú trọng sao cho không áp đặt người khác, chú ý cách diễn đạt sao cho không làm người đối thoại cảm thấy không thoải mái. Đây là qui tắc lịch sự đã được Lakoff (1977) đưa ra, theo Nguyễn Quang (2002:48) được coi là "lịch sự âm tính", chú trọng đến quyền lựa chọn của người nghe, để người nghe quyết định sự lựa chọn của mình. Qua phỏng vấn sau các hội thoại, chúng tôi cũng nhận thấy cả người Nhật và người Việt đều sử dụng aizuchi trong giao tiếp để thể hiện sự lịch sự, thể hiện sự quan tâm tới phát ngôn của người cùng tham gia hội thoại, tôn trọng người nghe. Trong nhiều trường hợp aizuchi được sử dụng để tránh đặt vấn đề một cách đột ngột, tránh nói thẳng trực tiếp vào ngay điều muốn nói. Trong văn hóa của nhiều nước Châu Á, cách nói đưa đẩy, không nói thẳng ngay vào vấn đề thường được coi là lối giao tiếp tế nhị, lịch sự. Ở nhiều văn cảnh giao tiếp, aizuchi tạo nên cách nói ngập ngừng, kéo dài thời gian, nhường quyền quyết định hoặc giảm nhẹ sự áp đặt cho người cùng tham gia đối thoại.

\subsection{Các loại aizuchi được sủe dụng}

Kết quả thống kê tần số sử dụng từng loại aizuchi của các nhóm khác nhau (Bảng 2) theo hình thức cho thấy, đối với cả người Nhật và người Việt, loại được sử dụng nhiều nhất là aizuchi với nghĩa hẹp, là các phát ngôn ngắn như うん, え, ええ, そう, はい, あのう，え えと, ねえ, まあね, じやあ, あら, へ え，なるほど，そうか，いいなあ。

Nhìn vào tỉ lệ của các loại aizuchi trong tổng số aizuchi của 2 nhóm $\mathrm{J}$ và $\mathrm{V}$, chúng ta thấy, người Nhật sử dụng P3- Diễn đạt bằng 
cách khác nhiều hơn người Việt, đặc biệt trong các tình huống khi người cùng đối thoại không hiểu hoặc nghe chưa rõ, người Nhật thường dùng cách diễn đạt khác nhiều hơn, trong khi người Việt sử dụng $\mathrm{P} 2$ - nhắc lại phát ngôn nhiều hơn. Kết quả này có thể một phần do yếu tố tâm lý là người bản ngữ thường có xu hướng tìm những từ ngữ và cách diễn đạt đơn giản hơn, dễ hiểu hơn khi giao tiếp với người phi bản ngữ, nhất là với những người phi bản ngữ mà trình độ tiếng còn chưa cao. Trong khảo sát này, những người tham gia hội thoại là người phi bản ngữ có trình độ tiếng Nhật từ N4, N3 đến N2, N1, trong đó chỉ có 1 người đạt N1. Liên quan đến trình độ người học tiếng Nhật và việc sử dụng aizuchi, 山本 (1992) đã nghiên cứu việc sử dụng aizuchi của người học tiếng Nhật trình độ sơ, trung, cao cấp. Tác giả rút ra kết luận rằng khi người học lên các trình độ cao hơn, tần số sử dụng aizuchi tiếng Nhật trong giao tiếp tăng lên, đồng thời loại aizuchi được sử dụng cũng phong phú hơn.

Trong hội thoại, có những khi người nghe không chờ người nói kết thúc phát ngôn, truyền đạt trọn vẹn đầy đủ thông tin, mà phán đoán trước nội dung phát ngôn của người nói và khi đang nghe giữa chừng đưa ra những từ ngữ chêm xen hay phát ngôn chêm xen đi trước (P4, P5). Ví dụ:

(K12)：J12：それは仕事にも難しい んで...

V12：関係ないですか、

J12：いや、関係ないんじゃなく $\tau, \ldots$

V12：うう。.

J12：子供もいろいろ時間があま りないので、. .
V12：そうですね。

Trong hội thoại trên đây, khi nghe J12 chia sẻ, V12 dự đoán trước ý mà J12 chưa nói và đưa ra phát ngôn đi trước関係ないですか. Đến lượt mình, J12 chỉnh sửa lại điều mà V12 dự đoánいや、関係ないんじゃなくて, sau đó tiếp tục phát ngôn định nói từ trước子供も いろいろ時間があまりないので.

Trong thực tế, không phải bao giờ người tham gia hội thoại cũng chờ cho đến khi đối tác dừng phát ngôn, hoặc kết thúc phát ngôn chêm xen rồi mới bắt đầu phát ngôn của mình. Phần chêm xen được đưa ra giữa phát ngôn của đối tác, diễn ra song song, đồng thời với phát ngôn của đối tác được quan sát thấy khá nhiều trong các hội thoại. Song nhìn Bảng 2 chúng ta thấy, aizuchi loại P4, P5 chiếm tỉ lệ không nhiều trong cả phát ngôn của người Nhật và người Việt, tỉ lệ này không có sự khác biệt đáng kể giữa người Nhật và người Việt. Để người nghe có thể phán đoán trước nội dung phát ngôn của người nói và nêu ra những phát ngôn như P4, P5, ít nhất, người nghe cần phải cùng có một nền kiến thức và những thông tin chung ở một mức độ nhất định với người nói, có sự quan tâm đến chủ đề hội thoại cùng những yếu tố khác nữa, trong đó, mối quan hệ thân sơ mà chúng tôi đề cập đến ở phần sau cũng là một yếu tố có ảnh hưởng đến việc lựa chọn hình thức phát ngôn.

\subsection{Chức năng của aizuchi}

Trong nghiên cứu này, chúng tôi thống kê aizuchi theo 5 nhóm chức năng dựa vào cách phân loại của堀口 (1997). Kết quả thống kê trên đây cho thấy, trong tất cả các cuộc hội thoại, aizuchi được dùng như một tín hiệu của người nghe, thể hiện rằng mình đang nghe chiếm tỉ lệ khá cao. Có thể nói, sử dụng aizuchi là một trong những biện pháp được sử 
dụng thường xuyên và thuận tiện để thể hiện mối quan tâm của người nghe đối với cuộc hội thoại và được coi là một trong những yếu tố lịch sự theo mức độ và qui chuẩn tùy vào các nền văn hóa xã hội khác nhau. Makino et al. (1997: 53) khi phác họa một "người nghe tích cực" đã mô tả "thay cho việc trao đổi ánh mắt, người Nhật dùng nhiều biện pháp khác để thể hiện rằng mình đang nghe", trong đó biện pháp tiêu biểu là aizuchi. Bằng việc truyền đạt tín hiệu đang nghe, người nghe cũng thể hiện rằng mình đang tham gia vào cuộc hội thoại, điều đó là vì người nghe và người nói có chung một nền hiểu biết nào đó.

Trong 5 nhóm chức năng, ở người Nhật, nhóm A chiếm tỉ lệ cao nhất, song chỉ là $44,5 \%$, thấp hơn nhiều so với $58,9 \%$ ở người Việt. Trừ chức năng $\mathrm{D}$ người Nhật và người Việt có tỉ lệ tần số sử dụng không chênh lệch đáng kể, các chức năng $\mathrm{B}, \mathrm{C}, \mathrm{E}$ người Nhật có tỉ lệ cao hơn. Liên quan đến chức năng của aizuchi trong giao tiếp, 水谷 (1983) đã gọi hội thoại kiểu Nhật là "đồng thoại”, trong sự so sánh với “đối thoại” kiểu Mỹ. Trong “đồng thoại", người nghe và người nói cùng hợp tác cho hội thoại tiến triển, trong đó aizuchi không chỉ những phát ngôn của người nói, mà cả aizuchi của người nghe cũng đóng vai trò tích cực cho hội thoại tiến triển.

\subsection{Yếu tố thân so và việc sử dụng aizuchi trong giao tiếp Nhật-Việt}

So sánh việc sử dụng aizuchi giữa những người có mức độ quan hệ thân thiết cao và những người mức độ thân thiết thấp, kết quả cho thấy, có sự chênh lệch về tần số sử dụng aizuchi giữa nhóm DH và DL ở cả người Nhật và người Việt. Như trên đã đề cập đến, việc sử dụng aizuchi được coi là một phương tiện thể hiện sự quan tâm của người nghe tới nội dung phát ngôn của người nói, sự tích cực tham gia vào cuộc hội thoại. Ở nhóm $\mathrm{DL}$, do mức độ thân sơ không cao, cả người Nhật và người Việt đều có xu hướng dùng aizuchi nhiều hơn như một phương tiện thể hiện sự quan tâm tới hội thoại, sự lịch sự trong giao tiếp.

Trong quá trình giao tiếp, không phải bao giờ người nói cũng nói ra tất cả mọi điều hay thể hiện được mọi điều mà mình muốn biểu đạt. Ví dụ, việc phủ nhận hay từ chối thẳng thừng ý kiến, quan điểm của người cùng tham gia hội thoại mà không giải thích gì thường là cách khó được chấp nhận trong mọi nền văn hóa, mọi ngôn ngữ. Tuy ở mỗi một ngôn ngữ, cách diễn đạt có thể có những mức độ trực tiếp hay gián tiếp khác nhau, theo những chuẩn mực xã hội khác nhau, song việc diễn đạt dài hơn, thêm các yếu tố "rườm" trong tiếng Nhật có xu hướng được sử dụng nhiều hơn, nhất là với các hội thoại giữa những người có mức độ thân quen không cao.

\section{Kết luận}

Nhật Bản và Việt Nam là hai quốc gia cùng nằm ở Châu Á và đều chịu ảnh hưởng mạnh mẽ của văn hóa chữ Hán, chính vì vậy hai dân tộc có nhiều nét tương đồng về văn hóa. Tuy nhiên, mỗi dân tộc lại có những quan niệm, những qui tắc ứng xử, những chuẩn mực văn hóa riêng của mình. Trên đây chúng tôi đã trình bày những kết quả nghiên cứu và chỉ ra sự khác biệt về tần số sử dụng cũng như loại aizuchi, những chức năng của aizuchi mà người Nhật và người Việt sử dụng trong giao tiếp Nhật-Việt. Aizuchi là một phương tiện quan trọng

Đối với việc dạy và học ngoại ngữ, việc nắm bắt khối lượng kiến thức ngôn ngữ về ngữ âm, từ vựng, ngữ pháp cần phải song song với hiểu biết những tri thức văn hóa ngôn ngữ. 
Người học một ngoại ngữ chỉ có thể thực sự sử dụng tốt ngôn ngữ thứ hai khi nhận thức được những đặc điểm văn hóa ngôn ngữ của người bản ngữ, hiểu được sự khác biệt giao văn hóa trong hoạt động giao tiếp. Trong tiếng Nhật, các aizuchi xuất hiện nhiều và là một trong những nguyên nhân khiến người học gặp khó khăn trong giao tiếp, đặc biệt trong nghe hiểu đối với người học ở trình độ sơ cấp và đầu trung cấp. Sự khác biệt trong việc sử dụng aizuchi cả về tần số cũng như ý nghĩa của aizuchi có thể là một nguyên nhân góp phần khiến cho người học không nghe được hay hiểu nhầm ý của phát ngôn. Việc đưa vào giới thiệu về các aizuchi và các ý nghĩa của chúng, các hình thức luyện tập cho người học, nhất là giai đoạn đầu là cần thiết và quan trọng và là một trong những giải pháp giúp người học khắc phục khó khăn trong nghe hiểu cũng như nâng cao khả năng khẩu ngữ cho người học.

Aizuchi là một phần quan trọng trong giao tiếp của người Nhật, có ảnh hưởng khá nhiều đến hiệu quả giao tiếp, còn khá nhiều vấn đề mà chúng tôi chưa thể tìm hiểu và trình bày hết trong phạm vi bài viết này. Nghiên cứu này gợi mở ra những hướng nghiên cứu triển vọng về giao tiếp giao văn hóa Nhật-Việt, đặc biệt là mối liên quan giữa việc sử dụng tiếng Nhật với các yếu tố như sự khác nhau về tuổi tác, giới tính, địa vị xã hội, môi trường làm việc thể hiện sự khác biệt về đặc trưng văn hóa giữa hai dân tộc. Trong những nghiên cứu tiếp theo, chúng tôi sẽ mở rộng đối tượng được khảo sát, tăng số lượng đối tượng ở các nhóm giả thiết có yếu tố ảnhh ưởng khác nhau vào tham gia hội thoại, đồng thời tìm hiểu sự khác biệt liên quan đến các yếu tố xã hội nêu trên.

Trong nghiên cứu này, mục đích mà chúng tôi đặt ra là xem xét đặc điểm sử dụng các aizuchi nhằm phát hiện và xác định những nét tương đồng và khác biệt về đặc điểm ngôn từ trong giao tiếp Nhật-Việt. Cùng với các yếu tố ngôn từ, các yếu tố phi ngôn từ cũng đóng vai trò rất quan trọng trong việc biểu đạt nội dung mà người tham gia hội thoại muốn truyền tải cũng như thể hiện các ý nghĩa tình thái. Trong những nghiên cứu khác chúng tôi sẽ đề cập đến những đặc điểm phi ngôn từ trong giao tiếp và xem xét sự khác biệt về những đặc điểm này giữa Nhật Bản và Việt Nam.

\section{Tài liệu tham khảo}

\section{Tiếng Việt}

Nguyễn Quang (2002). Giao tiếp và giao tiếp giao văn hóa. Hà Nội: NXB Đại học Quốc gia Hà Nội.

Hoàng Anh Thi (1997). Vài nét so sánh điểm khác biệt văn hoá Nhật Bản và Việt Nam thể hiện trong ngôn ngữ giao tiếp. Tạp chí Nghiên cúu Nhật Bản. số 1 năm 1997. tr. 44- 49.

Hoàng Anh Thi (2001). So sánh nghi thúc giao tiếp tiếng Nhật và tiếng Việt (qua tù̀ ngũ xung hô), Luận án tiến sỹ Ngữ văn, Trường ĐH KHXH\&NV, Hà Nội.

\section{Tiếng Anh}

Brown P. \& Levinson S. (1987). Politeness: Some Universals in Language Use. Cambridge: CUP.

Grice P. (1998). Studies in the Way of Words, Cambridge, MA.: Havard University Press (清塚邦彦訳『論理と会話』東京、勁草 書房).

John Hooker (2012). Cultural Differences in Business Communication. The Handbook of Intercultural Discourse and Communication, First Edition. Edited by Christina Bratt Paulston, Scott F. Kiesling, Elizabeth S. Rangel, Blackwell Publishing Ltd., pp. 389-407.

Julie Peters (2002). Discourse between Japanese and American Speakers: How Cross-cultural Communication Patterns Contribute to Misunderstandings. University of Michigan - Dearborn. 
Kenji Kitao, S. Kathleen Kitao, Joan Headrick Miller and Juliet Winters (1995). Culture and Communication. Yamaguti Shoten. Kyoto. Japan.

Lakoff, R. (1977) What You Can Do with Words: Politeness, Pragmatics and Perfomatives. In Rodger, Andy, Wall, Bob and Murphy, John (eds.), Proceedings of the Taxas Conference on Performatives, Presuppositions and Implicatures, 79-106. Arlington, V.A.: Center for Applied Linguistics.

Leech, G. N. (1983). Principles of Pragmatics. London, Longman.

Makino S., Hatasa Y., \& Hatasa K. (1997). Nakama: Japanese communication, culture, context, Houghton Mifflin, Boston.

Mark L. Knapp, Anita L. Vangelisti (1999). Interpersonal Communication and Human Relationship, Fourth Edition. Allyn and Bacon, Boston, zlondon, Toronto, Sydney, Tokyo, Singapore.

Steven Brown, Brenda Hayashi, and Kikue Yamamoto, Japan/ Anglo-American CrossCultural Communication (2012). The Handbook of Intercultural Discourse and Communication, First Edition. Edited by Christina Bratt Paulston, Scott F. Kiesling, Elizabeth S. Rangel, Blackwell Publishing Ltd., pp. 252-271.

Thomas M. Holgraves (2001). Language as Social Action: Social Psychology and Language Use. Psychology Press, New York.

Yamada, Haru (1997). Different Games, Different Rules. New York: Oxford University Press, New York.

\section{Tiếng Nhật}

今石幸子 (1992)。「談話における聞き手の 行動 - あいづちのタイミングについ て -」『日本語教育学会創立 30 周年・ 法人設立 15 周年記念大会予稿集』, pp. $147-151$.

大宮千鶴子 (1986)。「相づち使用の実態一出 現傾向とその周辺一」『語学研究論』 大東文化大学語学教育研究所.
大塚容子 (2005)。「テレビインタビュー番組 におけるあいづち的表現 - ポライトネ スの観点から - 」『岐阜聖徳学園大学 紀要(外国語学部編)』第44集, pp. 75 $-86$.

大塚容子 (2007)。「日本語母語話者の英語使 用場面におけるあいづち的表現一会話 管理ストラテジーの観点から」『岐阜 聖徳学園大学紀要 (外国語学部編)』第 46集, pp. 75-86.

大塚容子 (2009)。「母語話者と非母語話者 による会話におけるあいづち一日・英 語会話の比較」，『岐阜聖徳学園大学 紀要(外国語学部編)』第48集, pp. 95 $-106$

久保田真弓 (2001)。『「あいづち」は人を活 か寸』麇済堂出版.

黒崎良昭 (1987)。「談話進行上の相づちの運 用と機能 - 兵庫県滝野方言について 」『国語学』150号，pp. 109 - 122.

小宮千鶴子 (1986)。「相づち使用の実態 - 出 現傾向とその周辺 - 」『語学教育研究 諭』第3号, 大東文化大学語学教育研究 所, pp. 43-62.

陳姿葑 (2001)。「日本語の談話におけるあい づちの類型とその仕組み」『日本語教 育』108号，pp. 24-33.

陳姿莘 (2002)。「日本語におけるあいづち研 究の概観及びその展望」『言語文化と 日本語教育』2002年5月特集号.

堀口純子 (1997)。『日本語教育と会話分析』 くろしお出版.

松田陽子 (1988)。「対話の日本語教育学一あ いづちに関連してー」『日本語学』第7 巻，第13号，pp. 59-66.

水谷信子 (1983)。「あいづちと応答」水谷修 編『講座 日本語と表現3 話しことば の表現』，筑摩書房，pp. 37-44.

水谷信子 (1984)。「日本語教育と話し言葉の 実態 - あいづちの分析 -」『金田一春 彦博士稀記念論文集 第二巻 言語学 編』三省堂，pp. 261-279.

水谷信子 (1988)。「相づち諭」『日本語学』 第7巻，第13号明治書院，pp. 4-11.

村田晶子 (2000)。「学習者のあいづちの機能 分析一「聞いている」という信号，感 情・態度の表示，そして turn-taking に至るまでー」『世界の日本語教育』 ，第10号，pp. 241-260.

メイナード，泉子・K，『会話分析』くろし お出版，1993.

山本 美子 (1992)。「日本語学習者のあいづ 
ち使用実態の分析一頻度および種類一

$」$ 『言語文化と日本語教育』4号, pp. $22-34$.

楊 晶 (1997).「中国人学習者の日本語のあい づち使用に見られる母語からの影響一形 態、頻度、タイミングを中心に」『言語 文化と日本語教育』13号，pp. 117-128.

楊 晶 (1999)。「中・日両言語のあいづちに 関する一考察一頻度とその周辺一」『
人間文化研究年報』第 23 号, お茶の水女 子大学大学院人間文化研究科, pp. 28 -38 .

楊 晶（2001）。「電話会話で使用される中 国人学習者の日本語のあいづちについ て - 機能に着目した日本人との比較 -

」『日本語教育』第111号, pp. 46-55

\title{
BACK-CHANNELING EXPRESSIONS USED IN JAPANESE-VIETNAMESE COMMUNICATION FROM THE VIEWPOINT OF POLITENESS THEORY
}

\author{
Do Hoang Ngan \\ Faculty of Japanese Language and Culture, VNU University \\ and Languages and International Studies,
}

Pham Van Dong, Cau Giay, Hanoi, Vietnam

\begin{abstract}
This research aims at finding the linguistic culture similarities and differences between Japanese and Vietnamese in back-channeling expression using in Japanese-Vietnamese communication. The data collected from 12 conversations in pairs between a Japanese native speaker and a Vietnamese were analyzed by groups of aizuchi's types, functions and by relationship closureness between the conversation partners. The results show that both Japanese and Vietnamese use back-channeling words and short phrases the most often, but "Repetition" is used more often by Vietnamese while "Expression in a different way" is used more often by Japanese. Concerning the functions of backchannels, both Japanese and Vietnamese use backchannels as "Signal of listening" the most often, but Vietnamese use aizuchi with this function more often than Japanese, while Japanese use other functions more often than Vietnamese, except "Signal of disagreement" with no significant difference. On the other hand, the research shows that Japanese use backchannels more often despite the distance between conversation partners.
\end{abstract}

Keywords: Japanese-Vietnamese communication, linguistic culture, cross-cultural, politeness theory, backchannel 